\title{
Synergistic anti-hepatoma effect of bufalin combined with sorafenib via mediating the tumor vascular microenvironment by targeting mTOR/VEGF signaling
}

\author{
HAIYONG WANG ${ }^{1,2 *}$, CHENYUE ZHANG ${ }^{1,3 *}$, HUIYING $\mathrm{CHI}^{4}$ and ZHIQIANG MENG ${ }^{1,3}$ \\ ${ }^{1}$ Department of Integrative Oncology, Fudan University Shanghai Cancer Center, Shanghai 200032; ${ }^{2}$ Department of Internal \\ Medicine Oncology, Shandong Cancer Hospital and Institute, Shandong Cancer Hospital Affiliated to Shandong University, \\ Shandong Academy of Medical Sciences, Jinan, Shandong 250117; ${ }^{3}$ Department of Oncology, Shanghai Medical College, \\ Fudan University; ${ }^{4}$ Shanghai Geriatrics Institute of Traditional Chinese Medicine, Shanghai 200032, P.R. China
}

Received December 20, 2017; Accepted March 20, 2018

DOI: 10.3892/ijo.2018.4351

\begin{abstract}
Sorafenib inhibits tumor growth primarily by inhibiting vessel formation, however, its efficacy requires improvement, therefore, the development of strategies which augment its antiangiogenic effect are of primary concern. Bufalin inhibits tumor cell proliferation and metastasis, and induces apoptosis. In our previous study, it was demonstrated that the antiangiogenic effect of sorafenib was improved by bufalin in human umbilical vein endothelial cells (HUVECs). However, whether bufalin synergizes with sorafenib by affecting the tumor vascular microenvironment remains to be elucidated. In the present study, it was found that hepatocellular carcinoma (HCC) cell proliferation was inhibited by either bufalin or sorafenib following incubation for $24 \mathrm{~h}$, and the inhibition was enhanced upon treatment with a combination of the two. Conditioned medium (CM), comprising supernatant from $\mathrm{HCC}$ cells was collected from each of the treatment groups. The migration and tubule formation were suppressed the most in the combination-CM treated HUVECs. The secretion of vascular endothelial growth factor (VEGF) was decreased in $\mathrm{HCC}$ cells treated with the combination-CM, as determined by an angiogenesis array, enzyme-linked immunosorbent assay (ELISA) and western blot analysis. The inhibition of tube formation in HUVECs treated with the combination-CM was reversed by incubation with VEGF. The in vivo experiments demonstrated that subcutaneous HCC cell tumors from mice treated with the combination treatment expressed the lowest
\end{abstract}

Correspondence to: Professor Zhiqiang Meng, Department of Integrative Oncology, Fudan University Shanghai Cancer Center, 270 Dongan Road, Shanghai 200032, P.R. China

E-mail: mengzhq@yeah.net

"Contributed equally

Key words: bufalin, sorafenib, angiogenesis, hepatocellular carcinoma, mechanistic target of rapamycin/vascular endothelial growth factor signaling levels of VEGF, as evidenced by immunohistochemistry and ELISA. Additionally, the level of phosphorylated mechanistic target of rapamycin (mTOR) was reduced in HUVECs pretreated with the phosphoinositide 3-kinase inhibitor PI103. Furthermore, the migration of HCC cells and HUVEC tube formation were attenuated by PI103 pretreatment. In conclusion, the results revealed a synergistic anti-hepatoma effect of bufalin combined with sorafenib via affecting the tumor vascular microenvironment by targeting mTOR/VEGF signaling.

\section{Introduction}

Hepatocellular carcinoma (HCC) has been identified as a highly vascularized tumor $(1,2)$. Therefore, an effective option for the treatment of HCC is to suppress angiogenesis, as has been widely recognized and utilized by oncologists worldwide $(3,4)$. There have been numerous studies on tumor angiogenesis in previous years. For example, studies have revealed that the major cytokines responsible for tumor angiogenesis are secreted via the intricate interaction between tumor cells and stromal cells $(5,6)$. Among these factors, vascular endothelial growth factor (VEGF) is pivotal as the primary molecular driver in vasculogenesis (7). Inflammatory and tumor cells secrete VEGF, which binds and activates the VEGF receptor (VRGFR) on the surface of endothelial cells (ECs), stimulating various signaling pathways to accelerate angiogenesis (8-10). Therefore, the inhibition of tumor angiogenesis via the suppression of VEGF is favorably recommended by clinicians globally.

Sorafenib, a tyrosine kinase inhibitor, is recommended and widely used for patients with advanced HCC (11-13). However, its therapeutic effect in $\mathrm{HCC}$ is limited. Although it has been demonstrated to increase overall survival rates in patients with advanced stage HCC, the response rate is low, as resistance to and intolerance of sorafenib are common. It has been reported that pregnane $\mathrm{X}$ and proliferation and apoptosis adaptor protein 15 mediate sorafenib resistance $(14,15)$. Therefore, a combination of sorafenib with other treatment modalities may be advantageous. 
Bufalin, the major bioactive component isolated from toad venom, has been confirmed as a potent antitumor drug through its effect on tumor cell proliferation, apoptosis and migration (16-18). In our previous study, it was demonstrated that the antiangiogenic effect of sorafenib was markedly improved by combination with bufalin via targeting AKT/VEGF in human umbilical vein endothelial cells (HUVECs) and HCC cells (19). Bufalin was also demonstrated to suppress HCC metastasis via hypoxia inducible factor/VEGF signaling (20). Others reported the inhibitory effect of bufalin on HCC cell proliferation $(21,22)$. However, whether the synergistic effect of bufalin combined with sorafenib is achieved in HCC cells by targeting tumor cells and surrounding ECs remains to be elucidated, and the underlying mechanisms have not been identified. These issues were considered in the present study.

\section{Materials and methods}

Antibodies and reagents. The primary antibodies used in the present study included: VEGF (Bioworld Technology, Inc., St. Louis Park, MN, USA), phosphorylated AKT (p-AKT), $\mathrm{p}$-mechanistic target of rapamycin (p-mTOR), $\mathrm{p}$-phosphatase and tensin homolog (p-PTEN), p-extracellular signal-regulated kinase (p-ERK1/2), and AKT, mTOR, PTEN, ERK and GAPDH (Cell Signaling Technology, Inc., Danvers, MA, USA). The reagents used in the present study included: PI103 (Selleck Chemicals, Houston, TX, USA), the Human Angiogenesis Array Q1 (RayBiotech, Norcross, GA, USA), sorafenib (Selleck Chemicals) and bufalin (Sigma-Aldrich; Merck KGaA, Darmstadt, Germany)

Cell lines. The in vitro experiments were performed with HUVECs from the American Type Culture Collection (Manassas, VA, USA), and SMMC-7721 and PLC/PRF/5 HCC cells, which were purchased from the Cell Bank of Type Culture Collection of Chinese Academy of Sciences (Shanghai, China). The cells were propagated with DMEM containing $10 \%$ fetal bovine serum (FBS; HyClone, GE Healthcare Life Sciences, Logan, UT, USA) and $1 \%$ penicillin/streptomycin at $37^{\circ} \mathrm{C}$ in an atmosphere containing $5 \% \mathrm{CO}_{2}$.

Animals. Six-week old male Balb/c nude mice were used in the present study. The study was approved by Fudan University Shanghai Cancer Center (Shanghai, China). The mice were purchased from Vital River Laboratory Animal Technology Co., Ltd. (Beijing, China), and were raised under the following pathogen-free conditions: Room temperature, $20^{\circ} \mathrm{C}$; relative humidity, $\sim 50 \%$. The mice were given ad libitum access to food and water, and received humane care according to the principles of animal care of Fudan University (Shanghai, China). The mice were maintained under a 12-h light/dark cycle. The principles were as described in the Guide for the Care and Use of Laboratory Animals issued by the National Institutes of Health (Bethesda, MD, USA). The mice were randomly divided into four groups ( $\mathrm{n}=6$ mice/group). The mice in the experimental group were intraperitoneally injected with $1 \mathrm{mg} / \mathrm{kg}$ bufalin (5 days/week), oral uptake of $30 \mathrm{mg} / \mathrm{kg} / \mathrm{day}$ sorafenib (5 days/week), or combination treatment with the two drugs. The control mice were treated with the vehicle only. The treatment lasted for 16 days, following which the mice were sacrificed, and the tumors and blood were obtained. All experiments conformed to the ethical principles of animal experimentation stipulated by Fudan University.

In vivo tumorigenicity assay. A total of $5 \times 10^{6}$ SMMC-7721 cells in $0.2 \mathrm{ml}$ phosphate-buffered saline (PBS) were injected into the right flank of each mouse to form subcutaneous tumors. When the volume of these subcutaneous tumors reached a size of $100-300 \mathrm{~mm}^{3}$, the mice were treated with intraperitoneal injections of $\mathrm{mg} / \mathrm{kg}$ bufalin (5 days/week), $30 \mathrm{mg} / \mathrm{kg}$ oral sorafenib (5 days/week), or a combination of the two drugs. The control mice were treated with saline. Tumor size was measured every 4 days. The tumor-bearing mice were sacrificed following 16 days of treatments, and tumors and blood were extracted. The tumors were weighed and subjected to immunohistochemistry. All procedures conformed to the ethical principles of animal experimentation as stipulated by Fudan University.

Cell viability assay. To evaluate the effects of different treatments on cell viability, the HCC cells and HUVECs were plated in 96-well plates at a density of 5,000 cells per well. A total of $20 \mathrm{nM}$ bufalin, $10 \mu \mathrm{M}$ sorafenib, or a combination of the two were added to the wells and incubated at $37^{\circ} \mathrm{C}$ for 24 , 48 or 72 h. The cells were subjected to a Cell Counting Kit-8 assay (Dojindo Molecular Technologies, Inc., Kumamoto, Japan), used according to the manufacturer's protocol, and the absorbance was measured at a wavelength of $450 \mathrm{~nm}$ with a microplate reader to determine the cell viability rate.

Cell migration assay. To determine the effects of sorafenib, bufalin, or a combination of the two on cell migration, an assay was performed using Transwell inserts $(8-\mu \mathrm{m}$ pore; Corning Incorporated, Corning, NY, USA). The lower side of the chamber was filled with condition medium (CM) from the HCC cell lines (SMMC-7721 and PLC/PRF/5) following different treatments (control, bufalin, sorafenib, and combination). The HUVEC cells were plated on the upper side of the chamber. Following a 48-h incubation period, the migrated cells were fixed with methanol and stained with $0.1 \%$ crystal violet. The numbers of migrated cells were calculated in three different fields of view, at x20 magnification using an Olympus light microscope (Olympus Corporation, Tokyo, Japan).

Collection of CM. The SMMC7721 and PLC/PRF/5 cells were treated with bufalin, sorafenib, or the two in combination. At $48 \mathrm{~h}$ post-treatment, the cell supernatants were collected as $\mathrm{CM}$. The collected $\mathrm{CM}$ from each group was stored at $-80^{\circ} \mathrm{C}$ if not used immediately and was thawed prior to use.

Tube formation assay. A 96-well plate was coated with Matrigel and HUVEC cells were seeded at density of $4 \times 10^{4}$ cells per well in $100 \mu \mathrm{l} \mathrm{CM}$, with or without 1 or $6 \mathrm{ng} / \mathrm{ml}$ VEGF. The HUVECs were then incubated at $37^{\circ} \mathrm{C}$ for 12 , 24,36 or $48 \mathrm{~h}$. The central region of each well and the tube networks were analyzed using an Olympus light microscope (Olympus Corporation).

Detection of cytokines associated with angiogenesis using the human angiogenesis array kit. To determine the cytokines in 
A

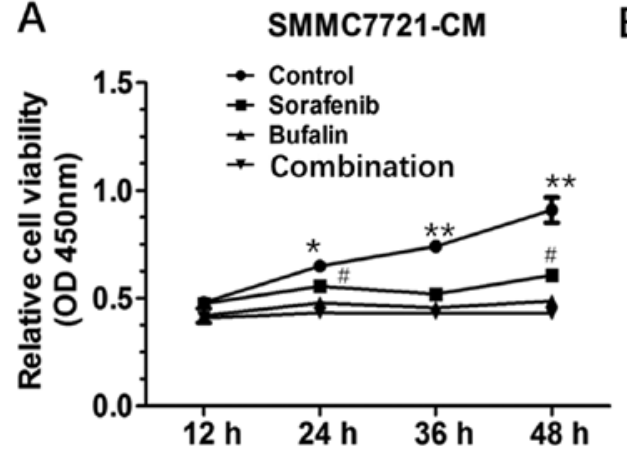

B

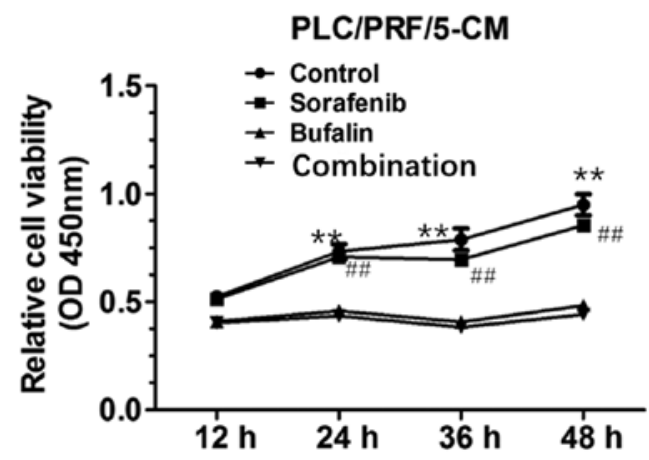

Figure 1. Bufalin enhances the inhibitory effect of sorafenib on hepatocellular carcinoma cell proliferation. (A) Viability rate of SMMC-7721 cells treated with $10 \mu \mathrm{M}$ sorafenib, $20 \mathrm{nM}$ bufalin, or the two in combination for 12, 24, 36 or $48 \mathrm{~h}$. (B) Viability rate of PLC/PRF/5 cells treated with $10 \mu \mathrm{M}$ sorafenib, $20 \mathrm{nM}$ bufalin or a combination for $12,24,36$ or $48 \mathrm{~h}$. ${ }^{*} \mathrm{P}<0.05$ control vs. combination; ${ }^{* *} \mathrm{P}<0.01$ control vs. combination; ${ }^{\#} \mathrm{P}<0.05$ sorafenib vs. combination; ${ }^{\# \#} \mathrm{P}<0.01$ sorafenib vs. combination.

SMMC-7721 cells treated with bufalin, sorafenib, or the two in combination, the Human Angiogenesis Array Q1 kit was used to detect angiogenesis-related cytokines in the $\mathrm{CM}$ extracted from HCC cells in the different treatment groups, according to the kit protocol. Following this, the VEGF concentration was determined using an enzyme-linked immunosorbent assay (ELISA) kit, according to the manufacturer's protocol and analyzed using a Labsystems Multiscan reader (Thermo Fisher Scientific, Inc., Waltham, MA, USA).

Western blot analysis. Western blot analysis was performed as previously described (23). Briefly, proteins were extracted from cells following the indicated treatments using radioimmunoprecipitation assay buffer (R0020; Beijing Solarbio, Science \& Technology Co., Ltd., Beijing, China). Protein concentrations were assessed using a bicinchoninic acid kit. Subsequently, protein samples $(12 \mu \mathrm{g})$ were loaded and separated by $10 \%$ SDS-PAGE for the detection of various proteins. Proteins were then transferred to polyvinylidene fluoride membranes, which were blocked in $5 \%$ skim milk for $1 \mathrm{~h}$ at $37^{\circ} \mathrm{C}$. The expression levels of VEGF, AKT, p-AKT, mTOR, pmTOR, PTEN and p-PTEN were detected by incubation with their respective primary antibodies overnight at $4^{\circ} \mathrm{C}$, after which the blots were washed three times with PBS. The primary antibodies used in the present study included: VEGF (20301555-1, 1:1,000; Bioworld Technology, Inc.), phosphorylated AKT (p-AKT; 4060, 1:2,000), p-mechanistic target of rapamycin (p-TOR; 5536, 1:1,000), p-phosphatase and tensin homolog (p-PTEN; 9551, 1:1,000), p-extracellular signalregulated kinase (p-ERK; 4370, 1:2,000), and AKT (4691, 1:1,000), m-TOR (2983, 1:1,000), PTEN (9188, 1:1,000), ERK (9102, 1:1,000) and GAPDH (5174, 1:1,000) (all from Cell Signaling Technology, Inc.). Finally, the blots were incubated with a horseradish peroxidase-conjugated immunoglobulin $\mathrm{G}$ secondary antibody (HAF008, 1:5,000; Novus Biologicals, LLC, Littleton, CO, USA) for $1 \mathrm{~h}$ at $37^{\circ} \mathrm{C}$, after which proteins were visualized by enhanced chemiluminescence (WBKLS0500; Merck KGaA). Semi-quantification of the blots was conducted using ImageJ software (version no. k 1.45; National Institutes of Health).

ELISA. The mice were treated with the different treatments, as described above. Blood samples were collected from the eyeballs of the mice. The expression of VEGF in the blood was measured using an ELISA kit, according to the manufacturer's protocol.

Immunohistochemistry. Immunohistochemistry protocols were performed as described previously (23). Samples were dehydrated in $70 \%$ ethanol, embedded in paraffin and sectioned (4 $\mu \mathrm{m})$. Briefly, the tumor sections were stained with rabbit anti-VEGF (1:1,000; cat. no. AP0742; Bioworld Technology, Inc.) at $4^{\circ} \mathrm{C}$ overnight, followed by a goat-anti-rabbit secondary antibody (1:1,000; cat. no. 7054; Cell Signaling Technology, Inc.) for $1 \mathrm{~h}$ at $37^{\circ} \mathrm{C}$. The expression of VEGF was calculated by multiplying the intensity score by the percentage score using an Olympus light microscope (Olympus Corporation). The staining intensity ( 0 , negative; 1 , weak; 2 , intense) and the ratio of stained cells $(0, \leq 10 \% ; 1,11-25 \% ; 2,26-50 \%$; $3,51-75 \% ; 4,>75 \%$ ) were evaluated by investigators blinded to the treatment status.

Statistical analysis. The results were analyzed using SPSS 22.0 software for Windows (IBM SPSS, Armonk, NY, USA). The comparisons between two groups were made using Student's t-test. Multi-group comparisons of the means were made using one-way analysis of variance with post hoc contrasts using the Student-Newman-Keuls test. All experiments were repeated three times. $\mathrm{P}<0.05$ was considered to indicate a statistically significant difference.

\section{Results}

Combination-CM induced the most marked inhibition of HUVEC proliferation. The SMMC-7721 and PLC/PRF/5 HCC cells were treated with $10 \mu \mathrm{M}$ sorafenib, $20 \mathrm{nM}$ bufalin, or a combination of the two for $12,24,36$ or $48 \mathrm{~h}$. The CM was collected $48 \mathrm{~h}$ following treatment. The HUVECs were subjected to CM from the HCC cells in the different treatment groups, and the proliferation of the HUVECs was evaluated. A CCK8 assay was used to determine whether a synergistic effect was present in the combination-treated HUVEC. The combination-CM led to a decrease in HUVEC proliferation from $24 \mathrm{~h}$, compared with that in the control-CM, bufalintreated $\mathrm{CM}$ and sorafenib-treated $\mathrm{CM}$. The effect of the combination-CM on HUVECs was most marked at $48 \mathrm{~h}$ 
A

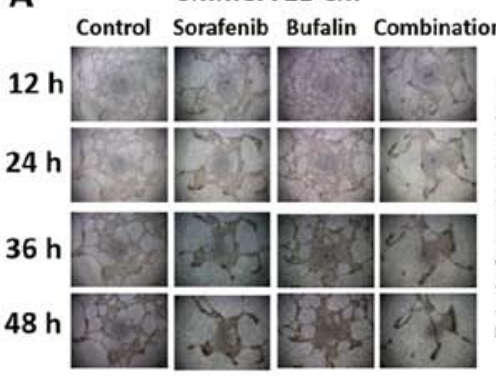

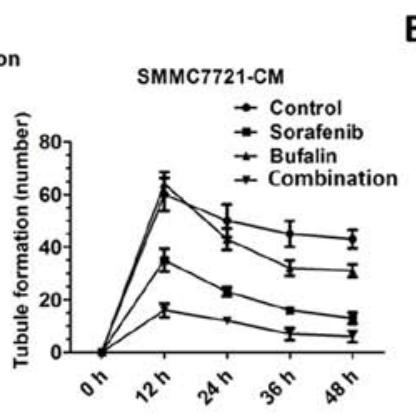

B PLC/PRF/5-CM

Control Sorafenib Bufalin Combination

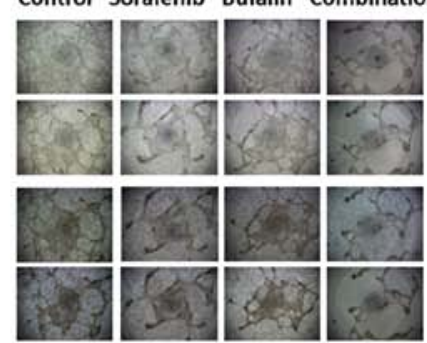

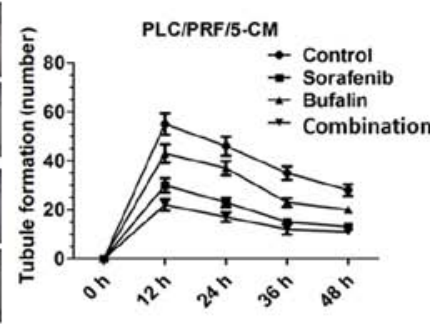

SMMC7721-CM
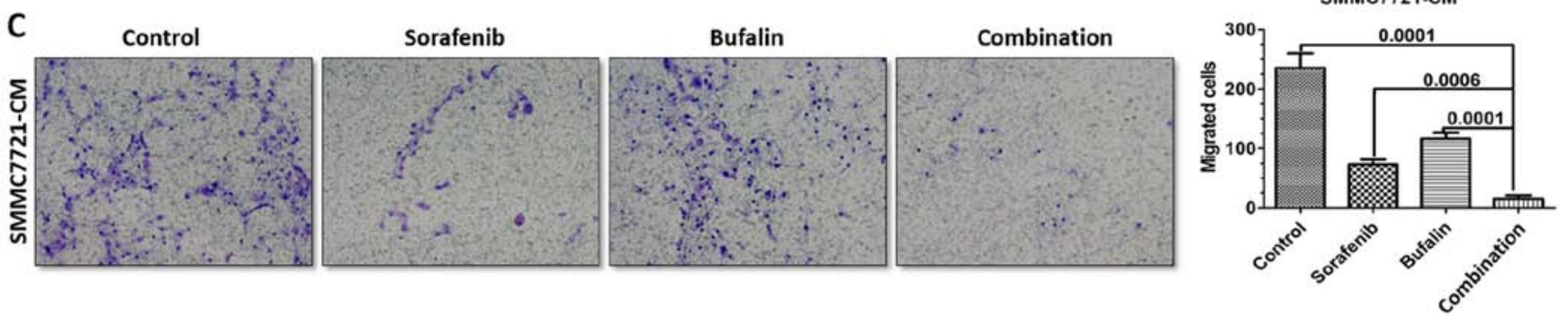

D

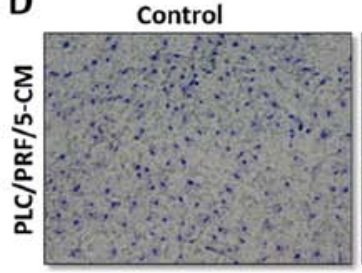

Sorafenib

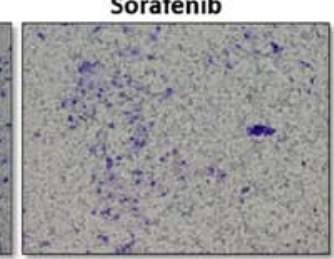

Bufalin

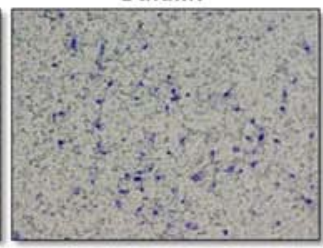

Combination

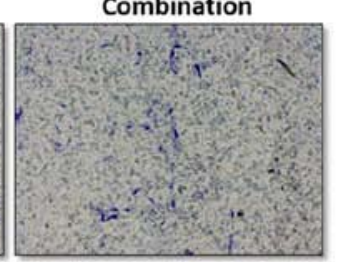

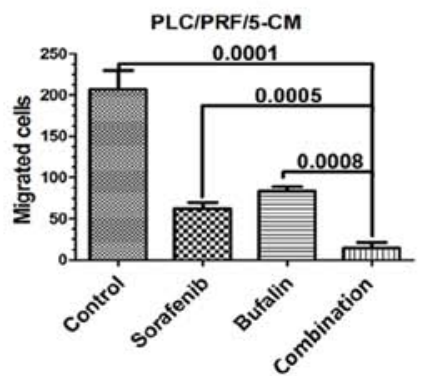

Figure 2. CM from cells receiving combined treatment results in an enhanced inhibitory effect on HUVEC tube formation in vitro. (A) Effects of SMMC-7721-CM from different treatment groups on HUVEC tube formation at 12, 24, 36 and $48 \mathrm{~h}$. (B) Effects of PLC/PRF/5-CM from different treatment groups on HUVEC tube formation at 12, 24, 36 and 48 h. (C) Representative images of migrated HUVECs treated with SMMC-7721-CM from a Transwell migration assay, and statistical analysis. (D) Representative images of migrated HUVECs treated with PLC/PRF/5-CM from a Transwell migration assay, and statistical analysis. CM, conditioned medium; HUVECs, human umbilical vein endothelial cells. (A and B) Magnification, x4; (C and D) magnification, x20.

post-incubation, compared with the control-CM and sorafenibtreated CM (Fig. 1).

Combination-treated CM inhibits HUVEC tube formation and migration. The SMMC-7721 and PLC/PRF/5 cells were incubated with $10 \mu \mathrm{M}$ sorafenib, $20 \mathrm{nM}$ bufalin, or a combination of the two. At 12, 24, 36 and $48 \mathrm{~h}$ post-treatment, the CM was collected and incubated with HUVECs. A tube formation assay was performed to observe the effect of the treatments. It was observed that the combination-CM inhibited the blood vessel formation of the HUVECs most markedly, compared with the other groups (Fig. 2A and B). The migration rates of the HUVECs in the different treatments groups were also detected. The migration rate of the HUVECs treated with the combination-CM was reduced compared with that of the HUVECs in the other treatment groups for the SMMC-7721 and PLC/PRF/5 cells (Fig. 2C and D).

Expression of VEGF is significantly reduced in combination-CM-treated HCC cells in vitro. As it was previously identified that combination-CM-treated HUVECs exhibited reduced blood vessel formation and inhibit migration, it was hypothesized that the expression of cytokines associated with angiogenesis may be altered in the CM from the combination-treated SMMC-7721 cells. Therefore, the CM from the untreated and combination-CM-treated SMMC7721 cells was analyzed using an angiogenesis-specific array for cytokine detection. Angiogenin, platelet-derived growth factor (PDGF-BB) and VEGF were altered most of the cytokines assessed, indicative of a potential role in facilitating angiogenesis (Fig. 3A). To further validate the alterations in cytokine expression detected by the human angiogenesis array, the concentration of VEGF was measured in the CM from SMMC-7721 and PLC/PRF/5 cells in the different treatment groups using ELISA (Fig. 3B and C). The results of the western blot analysis confirmed the ELISA results for the SMMC-7721 and PLC/PRF/5 cells (Fig. 3D).

$\mathrm{CM}$ from the SMMC7721 and PLC/PRF/5 cells collected at $48 \mathrm{~h}$ was added to HUVECs with or without the addition of 1 or $6 \mathrm{ng} / \mathrm{ml} \mathrm{VEGF,} \mathrm{and} \mathrm{tube} \mathrm{formation} \mathrm{was} \mathrm{observed.}$ The combination-CM suppressed HUVEC vessel formation. The incubation of HUVECs with VEGF disrupted the inhibitory effect on tubule formation induced by the addition of combination-CM. The higher concentration of VEGF resulted in enhanced vessel formation (Fig. 3E).

Combination treatment inhibits the secretion of VEGF in vivo. To measure the effect of the combination treatment on tumor growth in vivo, a subcutaneous tumor model was established. Mice received $1 \mathrm{mg} / \mathrm{kg} /$ day bufalin, $30 \mathrm{mg} / \mathrm{kg} /$ day 
A

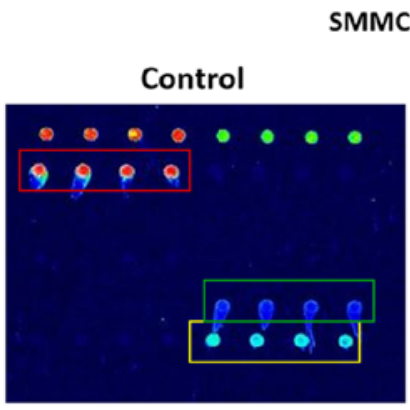

SMMC7721-CM

$\square$ Angiogenin $\square$ VEGF $\square$ PDGF-BB
Combination

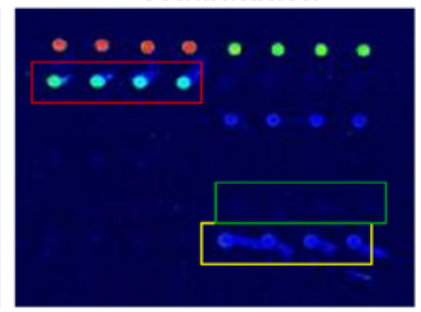

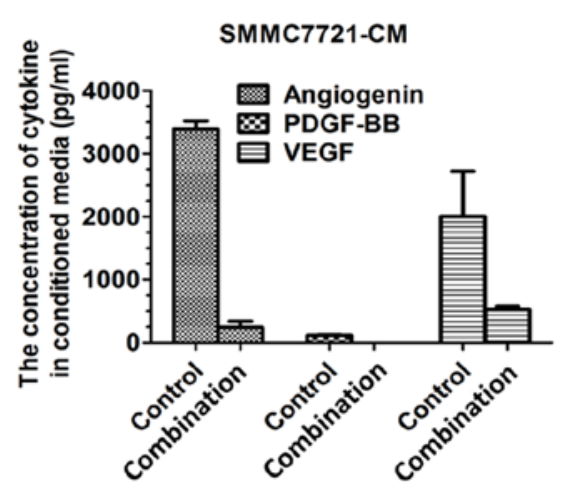

C
PLC/PRF/5-CM

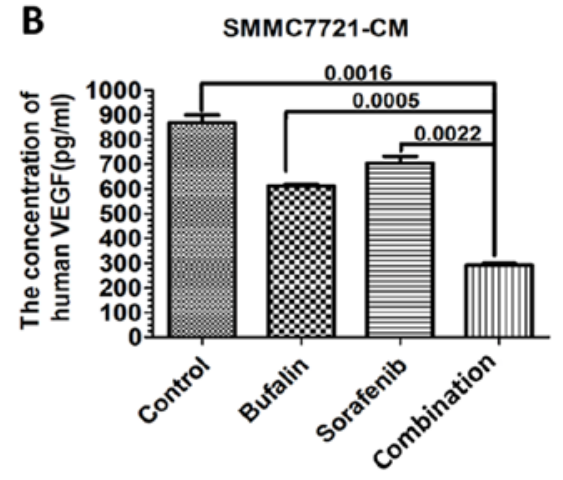

D

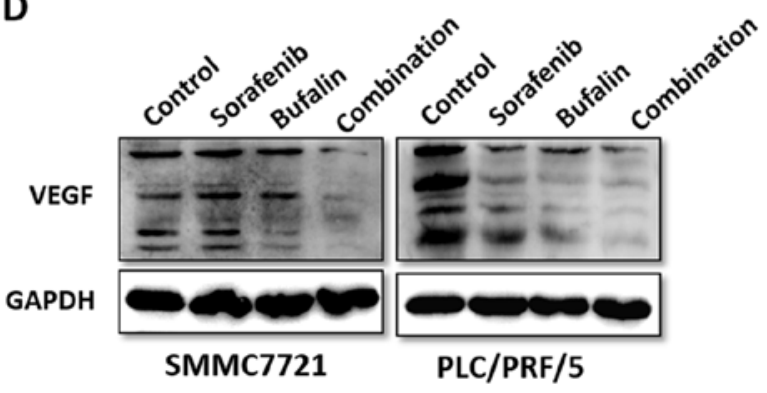

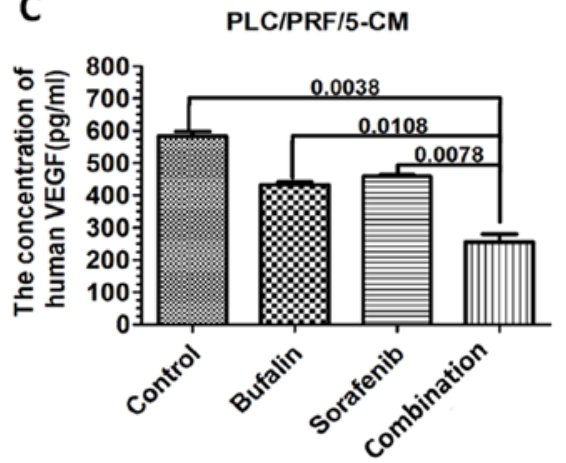

E

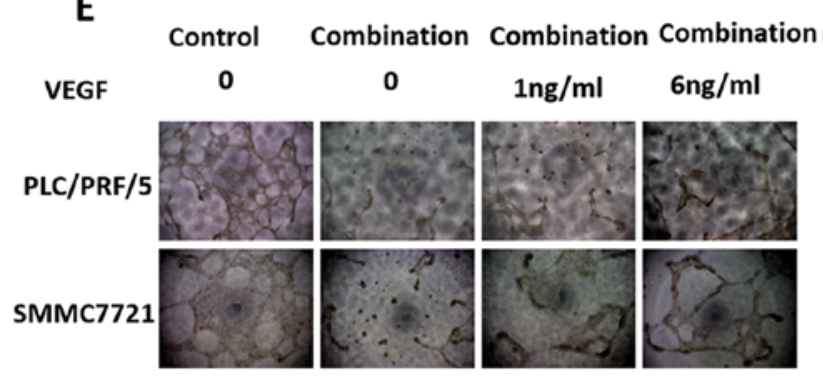

Figure 3. Expression of VEGF is significantly reduced in combination CM-treated HCC cells in vitro. (A) Measurement of angiogenesis-related cytokines using a Human Angiogenesis Array. The concentrations of angiogenin, PDGF-BB and VEGF were altered most. (B) Detection of secretion of VEGF in CM extracted from SMMC-7721 HCC cells in different treatment groups by ELISA. (C) Detection of secretion of VEGF in CM from PLC/PRF/5 HCC cells in different treatment groups by ELISA. (D) Protein expression of VEGF in SMMC-7721 and PLC/PRF/5 HCC cells in different treatment groups. (E) SMMC-7721 and PLC/PRF/5 HCC cells received the following treatments: Untreated; combination of sorafenib and bufalin; combination + VEGF (1 ng/ml); combination + VEGF (6 ng/ml); and CM was extracted. The effects of the CM on human umbilical vein endothelial cell tube formation are presented. Magnification, x4. VEGF, vascular endothelial growth factor; CM, conditioned medium; HCC, hepatocellular carcinoma; PDGF, platelet-derived growth factor; ELISA, enzyme-linked immunosorbent assay.

sorafenib, or a combination of the two for 16 days. The tumors were then excised and weighed. The results revealed that the subcutaneous tumors from mice that received the combination treatment were smaller, compared with those in the other groups (Fig. 4A). The tumor weights were significantly attenuated in mice that received the combination treatment, compared with those treated with bufalin or sorafenib alone (Fig. 4B). Further analyses of the expression of VEGF in tumors revealed that the expression of VEGF was lowest in the tumors and blood from mice that received the combination treatment, as determined with immunohistochemistry and ELISA (Fig. 4C and D).

Combination treatment affects HCC cells via the $m T O R / V E G F$ pathway. As it was identified that a decrease in the expression of VEGF occurred due to the combined treat- ment and that this may reduce the extent of angiogenesis in HCC, the pathways associated with VEGF were examined. The proteins upstream of VEGF were measured in HCC cells in the different treatments groups, including the phosphorylated and total levels of AKT, mTOR, PTEN and ERK, with western blot analysis. The HCC cells incubated with either bufalin or the combination treatment demonstrated a marginal decrease in the expression of $\mathrm{p}$-AKT. The expression of p-mTOR was also reduced in the HCC cells incubated with bufalin or the combination treatment. However, no significant alterations were identified in the phosphorylation of PTEN or ERK (Fig. 5A).

As a decrease was observed in the expression of $\mathrm{p}-\mathrm{AKT}$ and p-mTOR in the combination-treated SMMC-7721 cells, their involvement in the combination-induced reduction of VEGF was examined.PI103 $(2 \mu \mathrm{M})$ was selected as a specific pharmacological 
A
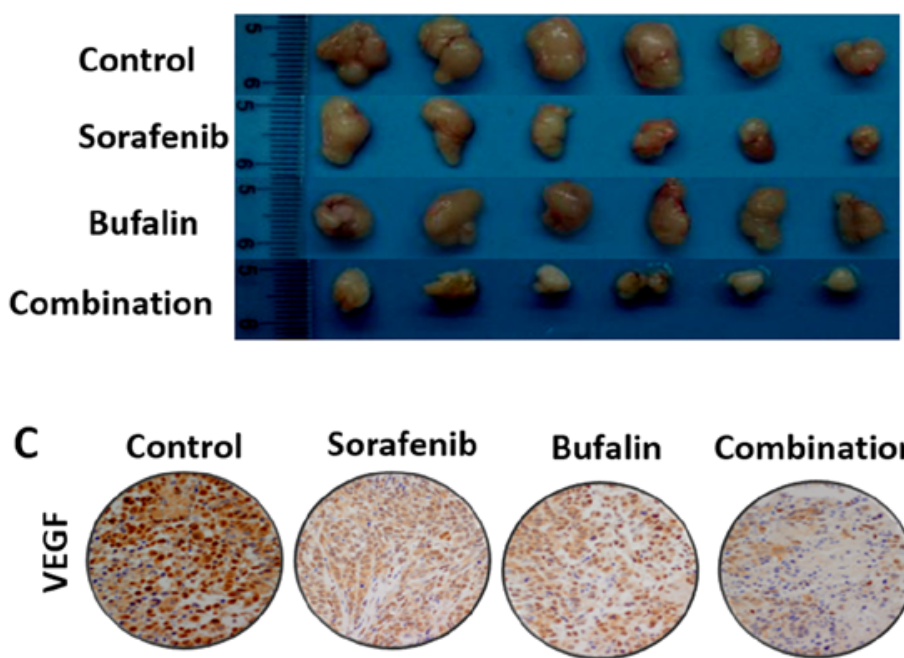
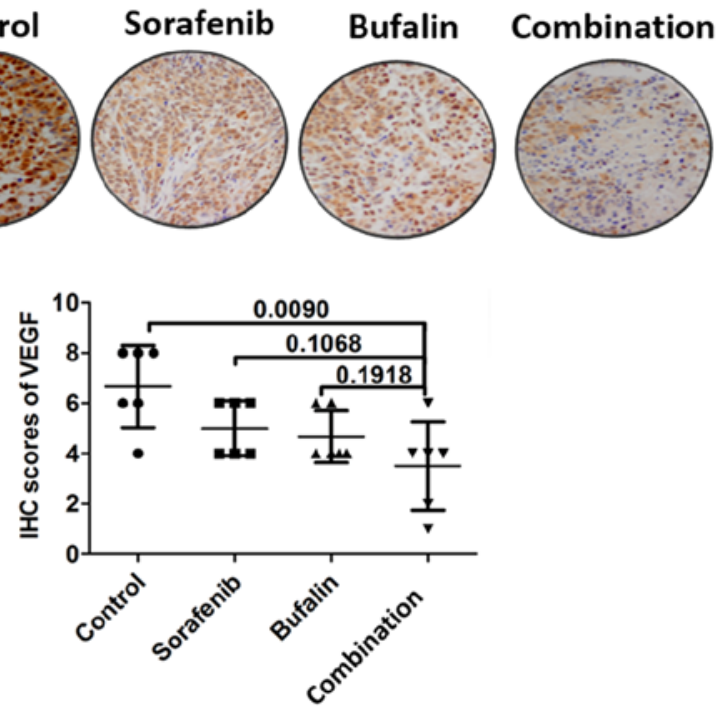

B
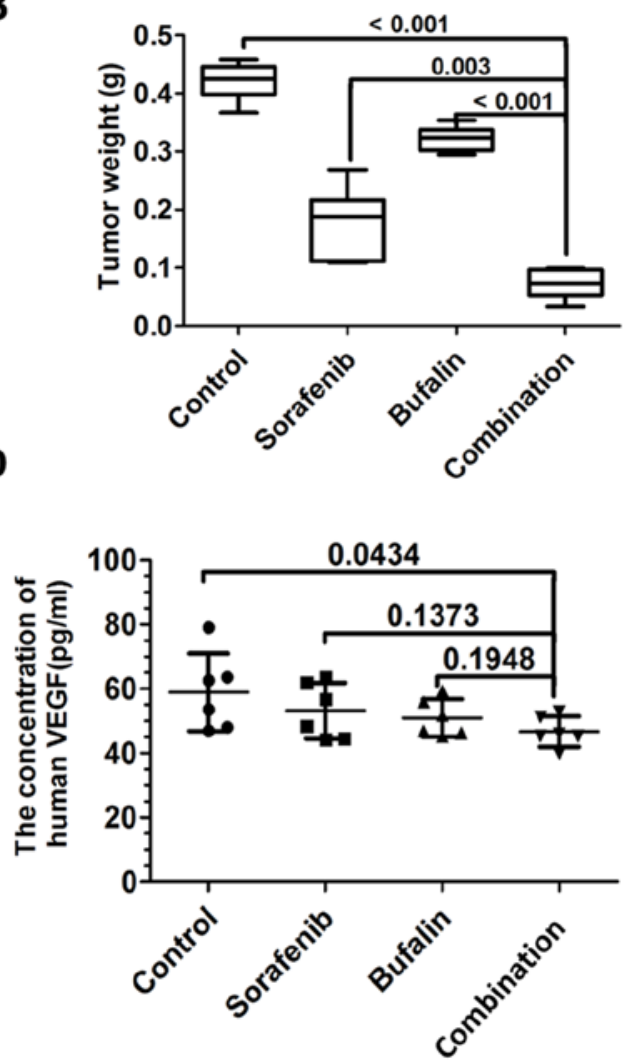

Figure 4. Combined treatment inhibits the expression of VEGF. (A) Orthotopic tumors of nude mice administered with $1 \mathrm{mg} / \mathrm{kg} / \mathrm{day}$ bufalin intraperitoneal injection, $30 \mathrm{mg} / \mathrm{kg} /$ day oral sorafenib, or a combination of the two for 14 days. (B) Tumor weights of the nude mice in different treatment groups (C) Representative IHC images of the expression of VEGF from nude mice administered with different treatments, as indicated, and the IHC scores for each group. Magnification, $x 40$. (D) Serum concentrations of VEGF were measured in nude mice in the different treatment groups. VEGF, vascular endothelial growth factor; IHC, immunohistochemistry.

inhibitor of the phosphoinositide 3-kinase (PI3K)/AKT/mTOR pathway. The results demonstrated that pretreatment with PI103 partially reversed the phosphorylation of mTOR (Fig. 5B). It was also observed that the expression of VEGF was significantly downregulated in the HCC cells pretreated with PI103, indicating the potential role of the mTOR pathway in modulating VEGF (Fig. 5C). The present study also evaluated the effect of the CM from cells pretreated with PI103 on HUVEC migration and tube formation. Compared with the CM from the non-pretreated cells, HUVEC migration and tube formation were suppressed by pretreatment with PI103 (Fig. 5D and E). Collectively, these results demonstrated that the mTOR pathway was involved in the reduced expression of VEGF induced by combination treatment.

Proposed mechanism by which bufalin augments the sorafenibinduced inhibition of angiogenesis through the mTOR/VEGF signaling pathway in HCC. The present study identified bufalin as asynergistic agent for HCC cells treated with sorafenib by regulating the $\mathrm{mTOR} / \mathrm{VEGF}$ pathway, leading to the reduced secretion of VEGF. The reduced binding of VEGF to VEGFR on the HUVECs led to reduced tumor angiogenesis. To the best of our knowledge, this is the first evidence of the synergistic anti-hepatoma effect of bufalin combined with sorafenib via the tumor vascular microenvironment by targeting mTOR/VEGF signaling (Fig. 6).

\section{Discussion}

Angiogenesis has been reported to be intrinsically linked with tumor growth, metastasis and development. Therefore, antiangiogenic cancer therapies have received much attention and have progressed rapidly $(2,24-27)$. As HCC is a highly vascularized tumor, inhibition of its angiogenesis by multiple drugs is considered a promising approach with therapeutic value.

Increasing evidence is available regarding the antitumor effect of bufalin on tumor cells. It has been reported to suppress proliferation and migration, and induce apoptosis in tumor cells (19-21). However, there are relatively few reports regarding the effect of bufalin on ECs. In our previous study, it was demonstrated that the synergistic effect of bufalin with sorafenib may be due to inhibition of the AKT/VEGF signaling pathway in HUVECs (22). Given the inhibitory effect of bufalin treatment on tumor cells and the established interactions between tumor cells and ECs, it was hypothesized that the synergistic role of bufalin with sorafenib may be partly explained by alterations to the cytokines in the tumor microenvironment, which affect the angiogenesis and migration of ECs.

To determine the effect of the combined treatment on HCC proliferation, two HCC cell lines (SMMC-7721 and $\mathrm{PLC} / \mathrm{PRF} / 5$ ) were used. The proliferation assay demonstrated 


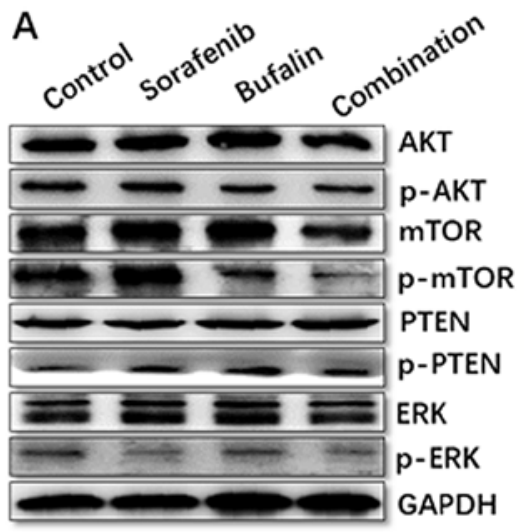

C

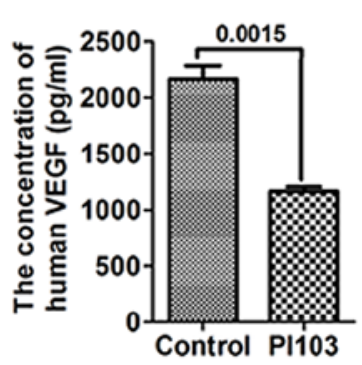

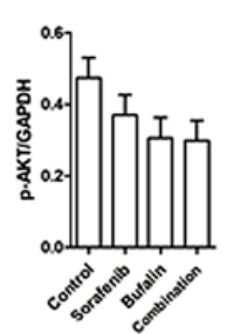

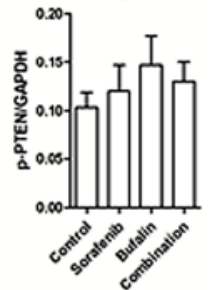

D

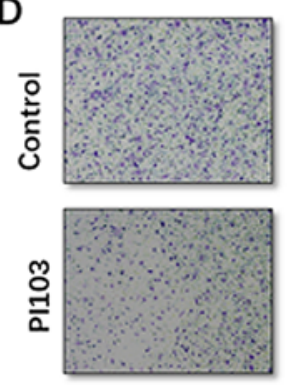

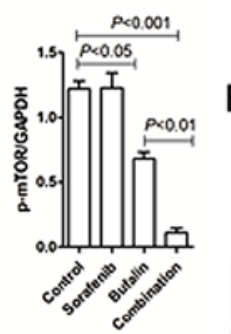

B
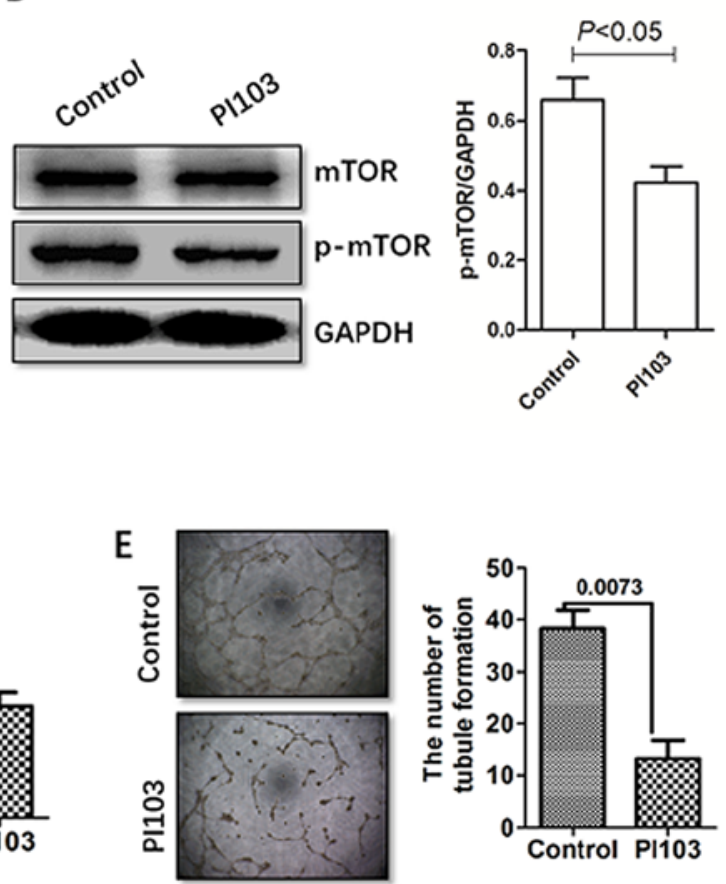

Figure 5. Combination CM regulates the secretion of VEGF via the mTOR pathway. (A) Expression levels of p-AKT, p-mTOR, p-PTEN and p-ERK were detected in HCC cells in different treatment groups by western blot analysis. (B) HCC cells were treated with the PI3K/AKT inhibitor PI103, followed by bufalin for $48 \mathrm{~h}$. The HCC cells were harvested, and p-mTOR and mTOR were detected by western blot analysis. (C) HCC cells were treated with the PI3K/AKT inhibitor PI103 $(2 \mu \mathrm{M})$. The secretion of VEGF was determined using an enzyme-linked immunosorbent assay. (D) HCC cells were treated with the PI3K/AKT inhibitor PI103. Representative images of the migrated HCC cells following incubation with CM derived from cells in the different treatment groups in the Transwell migration assay are presented and numbers shown. Magnification, x20. (E) Human umbilical vein endothelial cells were treated with a PI3K/AKT inhibitor PI103. Magnification, $\mathrm{x} 4$. Representative images of tubule formation following incubation in CM derived from different treatment groups, and analysis of the number of tubes formed is presented. CM, conditioned medium; VEGF, vascular endothelial growth factor; mTOR, mechanistic target of rapamycin; PTEN, phosphatase and tensin homolog; ERK, extracellular signal-regulated kinase; HCC, hepatocellular carcinoma; PI3K, phosphoinositide 3-kinase; p-, phosphorylated.

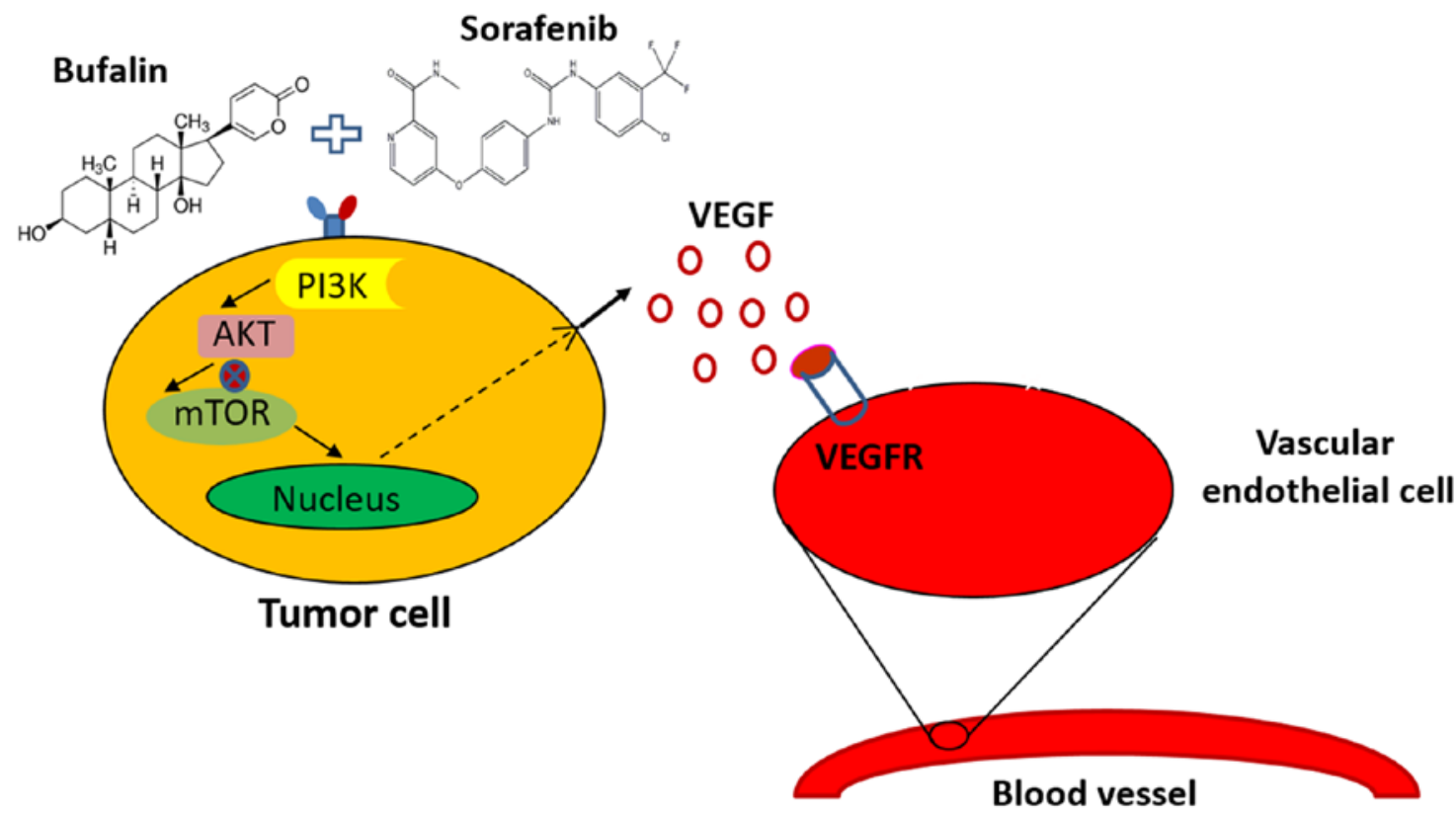

Figure 6. Diagram of the proposed mechanism by which bufalin augments the sorafenib-induced inhibition of angiogenesis through the mTOR/VEGF signaling pathway in HCC. The present study identified bufalin as producing synergistic effects with sorafenib against HCC by regulating the mTOR/VEGF pathway, leading to reduced VEGF secretion. Reduced VEGF binding to VEGFR on vascular endothelial cells led to reduced tumor angiogenesis. PI3K, phosphoinositide 3-kinase; mTOR, mechanistic target of rapamycin; HCC, hepatocellular carcinoma; VEGF, vascular endothelial growth factor; VEGFR, vascular endothelial growth factor receptor. 
that HCC cell viability was significantly reduced in HCC cells exposed to the combined treatment, compared with those treated with sorafenib alone. To test the hypothesis described above, CM from HCC cells in each of the different treatment groups was collected at $24 \mathrm{~h}$ post-incubation, and cultured with HUVECs for $12,24,36$ or $48 \mathrm{~h}$. As described in the results, the HUVECs incubated with the combination-CM underwent the least tube formation, suggesting there may be either less pro-angiogenic or more antiangiogenic cytokines produced by the HCC cells exposed to combination treatment. Furthermore, the number of migrated HUVECs was lowest following treatment with the combination-CM, as determined by a Transwell migration assay, which may be reflective of a metastasis-suppressive effect of the combined treatment. These results were in accord, highlighting the dual properties of combined treatment in the inhibition of endothelial angiogenesis and metastasis.

Subsequently, the present study aimed to ascertain the mechanisms that led to the attenuation of angiogenesis and metastasis in the HUVECs. Therefore, a human angiogenesis array was adopted. The results demonstrated that VEGF, PDGF-BB and angiogenin were the cytokines with the most marked significant alteration between the control cells and the cells exposed to the combined treatment. Of the three most altered cytokines, VEGF has been investigated most for its potent pro-angiogenic properties. VEGF initiates signaling pathways in ECs to promote proliferation, migration, survival and vascular permeability. VEGF inhibitors have been developed, as the overexpression of VEGF is frequently associated with a poorer prognosis in various types of cancer $(28,29)$. Subsequent ELISA and western blot analyses also confirmed the cytokine changes reported in the human angiogenesis array. To further corroborate that the inhibitory effect of combined treatment on the secretion of VEGF mediated the effect on angiogenesis, the HUVECs in the different treatment groups were treated with VEGF. The extent of vessel sprouting in the combination treatment HUVEC group was significantly reduced, which was reversed by incubation with VEGF. Therefore, the hypothesis was confirmed and validated.

The present study is notable as it focused not only on tumor cells, but also on ECs. The tumor microenvironment is a popular focus of investigations; the crosstalk between ECs and HCC cells can affect angiogenesis and metastasis via the regulation of inflammatory cytokines (30-32). The present study observed that the combination-CM derived from $\mathrm{HCC}$ cells attenuated angiogenesis and metastasis, indicating the potential of the drug combination in reducing the secretion of angiogenic cytokines.

In addition, in vivo experiments were performed to analyze the combined effect of bufalin and sorafenib. The expression of VEGF was reduced in the subcutaneous tumors and serum of mice receiving the combined treatment, compared with that in the mice receiving the drugs individually, as demonstrated by immunohistochemistry and ELISA. These results further demonstrated the synergistic role of bufalin and sorafenib in tumor inhibition via the suppression of VEGF.

There are numerous pathways involved in angiogenesis. For example, PI3K/AKT, PTEN/AKT, AKT/mTOR and mitogenactivated protein kinase kinase/ERK have all been associated with tumor angiogenesis via the upregulation of VEGF (33-36). Previous studies have demonstrated that the VEGF/PI3K/AKT pathway is associated with Nogo-B-induced angiogenesis in primary human retinal ECs (37). VEGF activates PI3K, which triggers the phosphorylation of AKT, leading to increased endothelial nitric oxide synthase activity. PI3K/AKT/mTOR is a pivotal signaling pathway responsible for cell proliferation, metabolism and motility $(38,39)$. Previous studies have also confirmed its involvement in angiogenesis $(40,41)$.

The present study determined the relative expression of the main proteins involved in the PI3K/AKT, PTEN/AKT, AKT/mTOR and MEK/ERK pathways. The results demonstrated that the level of p-AKT was significantly altered in the HCC cells that received the combined treatment, compared with that in the HCC cells treated with sorafenib alone. The level of p-m-TOR was decreased in the combination treatment group, compared with that in the SMMC-7721 cells treated with sorafenib. To further validate the role of the PI3K/AKT pathway in the altered expression of mTOR, the PI3K inhibitor PI103 was used. Incubation of the HCC cells with P1103 led to a marginal reduction of $\mathrm{p}-\mathrm{mTOR}$, whereas the application of PI103 led to a significant reduction in the expression of VEGF, reflecting the role of PI3K/AKT in the secretion of VEGF. This was also demonstrated in the migration and tube formation assays.

However, there were a number of limitations in the present study. First, the antiangiogenic effect of the combined drug was a comprehensive result of the balance between the production of fewer proangiogenic factors or increased secretion of antiangiogenic factors. There may be several other cytokine changes in the combination-treated CM, compared with the mono-drug-treated CM in addition to VEGF, however, the present study focused on VEGF only. Investigations are required to ascertain other angiogenic factor changes induced by the combination treatment in the future. Second, additional in vivo experiments are required to confirm the synergistic anti-hepatoma effect of the combined treatment of sorafenib and bufalin via mediating the tumor vascular microenvironment by targeting mTOR/VEGF signaling.

In conclusion, the present study identified bufalin as a synergistic agent for HCC treated with sorafenib by regulating tumor cells and ECs. The combined treatment affected HCC cells via the mTOR/VEGF pathway, leading to the reduced secretion of VEGF. The decreased secretion of VEGF produced by SMMC-7721 cells resulted in reduced migration and tube formation in HUVECs. Therefore, the mTOR/VEGF pathway may be one of the targets accounting for the enhanced effect of sorafenib treatment with bufalin.

\section{Acknowledgements}

Not applicable.

\section{Funding}

The present study was supported by the National Natural Science Foundation of China (grant nos. 81573753 and 81603348), the China Postdoctoral Fund (grant no. 21300075311104) and the Shandong Postdoctoral Innovation Special Fund (grant no. 201602012). 


\section{Availability of data and materials}

All data generated or analyzed during this study are included in this published article.

\section{Authors' contributions}

$\mathrm{HW}$ performed the in vivo studies and designed the study. $\mathrm{CZ}$ performed the in vitro studies and wrote the original manuscript. HC conducted the western blot experiments and analysed the data. ZM designed the study and reviewed the article.

\section{Ethics approval and consent to participate}

The study was approved by Fudan University Shanghai Cancer Center and all experiments conformed to the ethical principles of animal experimentation stipulated by Fudan University. The principles were as described in the Guide for the Care and Use of Laboratory Animals issued by the National Institutes of Health.

\section{Consent for publication}

Not applicable.

\section{Competing interests}

The authors declare that there are no competing interests.

\section{References}

1. Borzio M,Dionigi E,Parisi G, Raguzzi I and Sacco R: Management of hepatocellular carcinoma in the elderly. World J Hepatol 7: 1521-1529, 2015.

2. Folkman J: Tumor angiogenesis: Therapeutic implications. N Engl J Med 285: 1182-1186, 1971.

3. Folkman J: Anti-angiogenesis: New concept for therapy of solid tumors. Ann Surg 175: 409-416, 1972.

4. Folkman J: Role of angiogenesis in tumor growth and metastasis. Semin Oncol 29 (Suppl 16): 15-18, 2002.

5. Portillo-Lara R and Annabi N: Microengineered cancer-on-achip platforms to study the metastatic microenvironment. Lab Chip 16: 4063-4081, 2016.

6. Maj E, Papiernik D and Wietrzyk J: Antiangiogenic cancer treatment: The great discovery and greater complexity (Review). Int J Oncol 49: 1773-1784, 2016.

7. Yang Y, Zhang Y, Iwamoto H, Hosaka K, Seki T, Andersson P, Lim S, Fischer C, Nakamura M, Abe M, et al: Discontinuation of anti-VEGF cancer therapy promotes metastasis through a liver revascularization mechanism. Nat Commun 7: 12680, 2016.

8. Falchook GS, Moulder S, Naing A, Wheler JJ, Hong DS, Piha-Paul SA, Tsimberidou AM, Fu S, Zinner R, Janku F, et al: A phase I trial of combination trastuzumab, lapatinib, and bevacizumab in patients with advanced cancer. Invest New Drugs 33 177-186, 2015.

9. Fan X, Krieg S, Kuo CJ, Wiegand SJ, Rabinovitch M, Druzin ML, Brenner RM, Giudice LC and Nayak NR: VEGF blockade inhibits angiogenesis and reepithelialization of endometrium. FASEB J 22: 3571-3580, 2008.

10. Hong DS, Garrido-Laguna I,Ekmekcioglu S, Falchook GS, Naing A, Wheler JJ, Fu S, Moulder SL, Piha-Paul S, Tsimberidou AM, et al Dual inhibition of the vascular endothelial growth factor pathway: A phase 1 trial evaluating bevacizumab and AZD2171 (cediranib) in patients with advanced solid tumors. Cancer 120: 2164-2173, 2014.

11. Liu L, Cao Y, Chen C, Zhang X, McNabola A, Wilkie D, Wilhelm S, Lynch $M$ and Carter C: Sorafenib blocks the RAF/MEK/ERK pathway, inhibits tumor angiogenesis, and induces tumor cell apoptosis in hepatocellular carcinoma model PLC/PRF/5. Cancer Res 66: 11851-11858, 2006.
12. Pressiani T, Boni C, Rimassa L, Labianca R, Fagiuoli S, Salvagni S, Ferrari D, Cortesi E, Porta C, Mucciarini C, et al: Sorafenib in patients with Child-Pugh class A and B advanced hepatocellular carcinoma: A prospective feasibility analysis. Ann Oncol 24: 406-411, 2013.

13. Sposito C, Mariani L, Germini A, Flores Reyes M, Bongini M, Grossi G, Bhoori S and Mazzaferro V: Comparative efficacy of sorafenib versus best supportive care in recurrent hepatocellular carcinoma after liver transplantation: A case-control study. J Hepatol 59: 59-66, 2013.

14. Feng F, Jiang Q, Cao S, Cao Y, Li R, Shen L, Zhu H, Wang T, Sun L, Liang E, et al: Pregnane $\mathrm{X}$ receptor mediates sorafenib resistance in advanced hepatocellular carcinoma. Biochim Biophys Acta 1862: 1017-1030, 2018.

15. Quintavalle C, Hindupur SK, Quagliata L, Pallante P, Nigro C, Condorelli G, Andersen JB, Tagscherer KE, Roth W, Beguinot F, et al: Phosphoprotein enriched in diabetes (PED/PEA15) promotes migration in hepatocellular carcinoma and confers resistance to sorafenib. Cell Death Dis 8: e3138, 2017.

16. Takai N, Ueda T, Nishida M, Nasu K and Narahara H: Bufalin induces growth inhibition, cell cycle arrest and apoptosis in human endometrial and ovarian cancer cells. Int J Mol Med 21: 637-643, 2008.

17. Huang WW, Yang JS, Pai SJ, Wu PP, Chang SJ, Chueh FS, Fan MJ, Chiou SM, Kuo HM, Yeh CC, et al: Bufalin induces G0/G1 phase arrest through inhibiting the levels of cyclin D, cyclin E, CDK2 and CDK4, and triggers apoptosis via mitochondrial signaling pathway in T24 human bladder cancer cells. Mutat Res 732: 26-33, 2012 .

18. Yin JQ, Shen JN, Su WW, Wang J, Huang G, Jin S, Guo QC, Zou CY, Li HM and Li FB: Bufalin induces apoptosis in human osteosarcoma U-2OS and U-2OS methotrexate300-resistant cell lines. Acta Pharmacol Sin 28: 712-720, 2007.

19. Wang H, Zhang C, Ning Z, Xu L, Zhu X and Meng Z: Bufalin enhances anti-angiogenic effect of sorafenib via AKT/VEGF signaling. Int J Oncol 48: 1229-1241, 2016.

20. Wang H, Zhang C, Xu L, Zang K, Ning Z, Jiang F, Chi H, Zhu X and Meng Z: Bufalin suppresses hepatocellular carcinoma invasion and metastasis by targeting HIF- $1 \alpha$ via the PI3K/AKT/ mTOR pathway. Oncotarget 7: 20193-20208, 2016.

21. Qiu DZ, Zhang ZJ, Wu WZ and Yang YK: Bufalin, a component in Chansu, inhibits proliferation and invasion of hepatocellular carcinoma cells. BMC Complement Altern Med 13: 185, 2013.

22. Gao Y, Li HX, Xu LT, Wang P, Xu LY, Cohen L, Yang PY, $\mathrm{Gu} \mathrm{K}$ and Meng ZQ: Bufalin enhances the anti-proliferative effect of sorafenib on human hepatocellular carcinoma cells through downregulation of ERK. Mol Biol Rep 39: 1683-1689, 2012.

23. Zhuang L, Xu L, Wang P, Jiang Y, Yong P, Zhang C, Zhang H, Meng $Z$ and Yang $P$ : $\mathrm{Na}^{+} / \mathrm{K}^{+}$-ATPase $\alpha 1$ subunit, a novel therapeutic target for hepatocellular carcinoma. Oncotarget 6: 28183-28193, 2015.

24. Butler JM, Kobayashi H and Rafii S: Instructive role of the vascular niche in promoting tumour growth and tissue repair by angiocrine factors. Nat Rev Cancer 10: 138-146, 2010.

25. Kerbel RS: Tumor angiogenesis. N Engl J Med 358: 2039-2049, 2008.

26. Ferrara N and Kerbel RS: Angiogenesis as a therapeutic target. Nature 438: 967-974, 2005.

27. Bridges EM and Harris AL: The angiogenic process as a therapeutic target in cancer. Biochem Pharmacol 81: 1183-1191, 2011.

28. Fu L, Shi K, Wang J, Chen W, Shi D, Tian Y, Guo W, Yu W, Xiao X, Kang T, et al: TFAP2B overexpression contributes to tumor growth and a poor prognosis of human lung adenocarcinoma through modulation of ERK and VEGF/PEDF signaling. Mol Cancer 13: 89, 2014.

29. Chen C, Chi H, Min L and Junhua Z: Downregulation of guanine nucleotide-binding protein beta 1 (GNB1) is associated with worsened prognosis of clearcell renal cell carcinoma and is related to VEGF signaling pathway. J BUON 22: 1441-1446, 2017.

30. Neiva KG, Zhang Z, Miyazawa M, Warner KA, Karl E and Nör JE: Cross talk initiated by endothelial cells enhances migration and inhibits anoikis of squamous cell carcinoma cells through STAT3/ Akt/ERK signaling. Neoplasia 11: 583-593, 2009.

31. Warner KA, Miyazawa M, Cordeiro MM, Love WJ, Pinsky MS, Neiva KG, Spalding AC and Nör JE: Endothelial cells enhance tumor cell invasion through a crosstalk mediated by CXC chemokine signaling. Neoplasia 10: 131-139, 2008. 
32. ManziM,BacigalupoML,CarabiasP,ElolaMT,Wolfenstein-TodelC, Rabinovich GA, Espelt MV and Troncoso MF: Galectin-1 controls the proliferation and migration of liver sinusoidal endothelial cells and their interaction with hepatocarcinoma cells. J Cell Physiol 231: 1522-1533, 2016.

33. Liu WL, Gao M, Tzen KY, Tsai CL, Hsu FM, Cheng AL and Cheng JC: Targeting phosphatidylinositide 3-kinase/Akt pathway by BKM120 for radiosensitization in hepatocellular carcinoma. Oncotarget 5: 3662-3672, 2014.

34. Paplomata E and O'Regan R: The PI3K/AKT/mTOR pathway in breast cancer: Targets, trials and biomarkers. Ther Adv Med Oncol 6: 154-166, 2014.

35. Lee MS, Jeong MH, Lee HW, Han HJ, Ko A, Hewitt SM, Kim JH, Chun KH, Chung JY, Lee C, et al: PI3K/AKT activation induces PTEN ubiquitination and destabilization accelerating tumourigenesis. Nat Commun 6: 7769, 2015.

36. Zhang D, Li X, Yao Z, Wei C, Ning $N$ and Li J: GABAergic signaling facilitates breast cancer metastasis by promoting ERK1/2 dependent phosphorylation. Cancer Lett 348: 100-108, 2014.

37. Zhang Y, Wang L, Zhang Y, Wang M, Sun Q, Xia F, Wang R and Liu L: Nogo-B promotes angiogenesis in proliferative diabetic retinopathy via VEGF/PI3K/Akt pathway in an autocrine manner. Cell Physiol Biochem 43: 1742-1754, 2017.
38. Philippova M, Joshi MB, Pfaff D, Kyriakakis E, Maslova K, Erne P and Resink TJ: T-cadherin attenuates insulin-dependent signalling, eNOS activation, and angiogenesis in vascular endothelial cells. Cardiovasc Res 93: 498-507, 2012.

39. Sharma S, Guru SK, Manda S, Kumar A, Mintoo MJ, Prasad VD, Sharma PR, Mondhe DM, Bharate SB and Bhushan S: A marine sponge alkaloid derivative 4-chloro fascaplysin inhibits tumor growth and VEGF mediated angiogenesis by disrupting PI3K/Akt/ mTOR signaling cascade. Chem Biol Interact 275: 47-60, 2017.

40. Zhang W, Xiong Z, Wei T, Li Q, Tan Y, Ling L and Feng X: Nuclear factor 90 promotes angiogenesis by regulating HIF-1 $\alpha /$ VEGF-A expression through the PI3K/Akt signaling pathway in human cervical cancer. Cell Death Dis 9: 276, 2018.

41. Song ZY, Wang F, Cui SX and Qu XJ: Knockdown of CXCR4 inhibits CXCL12-induced angiogenesis in HUVECs through downregulation of the MAPK/ERK and PI3K/AKT and the Wnt// -catenin pathways. Cancer Invest 36: 10-18, 2018. 